\title{
Wavefront measurements of imaging systems by comparing a Point-Diffraction Interferometer and a Shack-Hartmann wavefront sensor
}

\author{
Medida del frente de onda en sistemas formadores de imagen \\ comparando un Interferómetro de Difracción por Punto y un sensor \\ de frentes de onda Shack-Hartmann
}

\author{
A. Marzoa $1 S^{*}$, S. Vallmitjana1S and S. Bosch ${ }^{1 \mathrm{~S}}$ \\ 1. Departament de Física Aplicada, Facultat de Física, Universidad de Barcelona, \\ 08028 Barcelona, (Spain)
}

(*) E-mail: tony.marzoa@ub.edu

S: SEDOPTICA member

Received: 30/03/2018 Accepted: 22/06/2018

DOI: $10.7149 /$ OPA.51.2.50027

\begin{abstract}
:
In this work, we present a comparative study between two notorious techniques for wavefront characterization: Shack-Hartmann wavefront sensor and the Point-Diffraction Interferometer. The main goal of this work is to experimentally demonstrate the equivalence of results when wavefront characterization measurements are carried out for both techniques, within the experimental error, in the case of imaging systems. The study has been carried out in several commercial convergent lenses obtaining small root-mean-square difference between both techniques. The results aim the possibility to design a dual set-up combining both techniques with potential use in Ophthalmic applications.
\end{abstract}

Key words: Optical Metrology, Wavefront, Aberrations, Shack-Hartmann, Interferometry

\section{RESUMEN:}

En este trabajo se describen dos técnicas para la caracterización del frente de onda en sistemas ópticos. Se trata del sensor de frentes de onda Shack-Hartmann y del Interferómetro de Difracción por Punto. El objetivo principal de este trabajo es probar experimentalmente la equivalencia entre ambas técnicas, dentro del error experimental, en la caracterización de sistemas formadores de imagen. El estudio se ha realizado en el caso de distintas lentes convergentes de tipo comercial. Los resultados abren la posibilidad de diseñar un sistema dual que integre ambas técnicas, con potencial aplicación en Óptica Oftálmica.

Palabras clave: Metrología Óptica, Frente de Onda, Aberraciones, Shack-Hartmann, Interferometria

\section{REFERENCES AND LINKS / REFERENCIAS Y ENLACES}

[1] Smith W J, 2000 Modern optical engineering (New York: McGraw-Hill)

[2] Born M and Wolf E, 2001 Principles of Optics (Cambridge University Press)

[3] Mahajan V N, 2011 Aberration Theory Made Simple (Bellingham: SPIE Press)

[4] Zernike F, 1934 Beugungstheorie des Schneidenverfahrens und Seiner Verbesserten Form, der Phasenkontrastmethode Physica 1 (8) 689-704

[5] Lakshminarayanan V and Fleck A, 2011 Zernike polynomials: a guide Journal of Modern Optics 58 (7) 545-561

[6] Noll R J, 1976 Zernike polynomials and atmospheric turbulence J. Opt. Soc. Am. 66 (3) 207-2011 
[7] Platt B C, 2001 History and principles of Shack-Hartmann wavefront sensing, J. Refract. Surg. 17 573577

[8] Duffner R W and Fugate R Q, 2009 The Adaptive Optics Revolution: A History (University of New Mexico Press)

[9] Liang J, Grimm B, Goelz S and Bille J F, 1994 Objective measurement of wave aberrations of the human eye with the use of a Hartmann-Shack wave-front sensor J. Opt. Soc. Am. 11 (7) 1949-1957

[10] Rodrigo P J, Eriksen R L, Daria V R and Glückstad J, 2003 Shack-Hartmann multiple-beam optical tweezers Opt. Express 11 (3) 208-214

[11] López-Quesada C, Andilla J and Martín-Badosa E, 2009 Correction of aberration in holographic optical tweezers using Shack-Hartmann sensor App. Optics 48 (6) 1084-1090

[12] Linnik W P, 1933 A Simple Interferometer for the Investigation of Optical Systems C. R. Acad. Sci, URSS $5(210)$

[13] Smartt R N and Steel W H, 1975 Theory and Application of Point-Diffraction Interferometers Japan J. Appl. Phys. 14 (1) 351-355

[14] Acosta E, Chamadoira S and Blendowske R, 2006 Modified point diffraction interferometer for inspection and evaluation of ophthalmic components J. Opt. Soc. Am. 23 (3) 632-637

[15] Chamadoira S, 2011 Interferómetro de difracción por orificio, IDO, para la inspección y caracterización de lentes progresivas (Universidae de Santiago de Compostela, PhD. Thesis)

[16] Vallmitjana S, Ricart I, Bosch S, Gargallo A and Acosta E, 2015 Point diffraction interferometry to measure local curvatures and caustics of noisy wave fronts: Application for determining optical properties of fish lenses J. Eur. Opt. Soc.-Rapid 1015010

[17] Abbe E, 1873 Beiträge zur Theorie des Mikroskops un der Mikroskopischen wahrnehmung Archiv für Mikroskopische Anatomie 9 413-468

[18] Rayleigh L, 1896 On the theory of optical images, with special references to the microscope Phil. Mag. $46(5), 167$

[19] Atmosfringe: http://www.atmossoftware.it/AtmosFringe.html

[20] Bará S, Pailos E and Arines J, 2012 Signal-to-noise ratio and aberration statistics in ocular aberrometry Optics Letters 37 (12), 2427-2429

[21] Bueno J M, Acosta E, Schwarz C and Artal P, 2009 Wavefront measurements of phase plates combining a point-diffraction interferometer and a Hartmann-Shack sensor Applied Optics 49 (3)

[22] Huang C, Raasch T W, Yi A Y, Sheedy J E, Andre B and Bullimore M A, 2012 Comparison of Three Techniques in Measuring Progressive Addition Lenses Optometry and Vision Science 89 (11) 15641573

[23] Alba-Bueno F, Millán M S and Vega F, 2017 Optical Characterization of Intraocular Lenses Opt. Pura Apl. 50 (1) 63-73

\section{Introduction}

It is a well-known fact from Geometrical Optics that, knowing the geometrical (curvature radii) and optical (refractive index) parameters of the elements that compose an optical system it is possible to determinate the position and size of the image of an object seen through this system [1]. If the optical system has rotational symmetry and the conditions of paraxial optics are respected, it is possible to obtain a perfect optical representation between pairs of points. These conditions are very restrictive in real optical systems, and then the image starts to present some defects that are known as optical aberrations (or geometrical aberrations).

Since optical imaging systems has turned into a fundamental tool in different areas, such as science and media, in our society, it seems reasonable and interesting to analyze and quantify those aberrations, in order to compensate it and to improve the quality of the optical instrument under test.

In that sense, many different techniques and methods where developed during the last decades. Within this large set of techniques, there are two relevant and widely used methods. This is the case of Shack-Hartmann (SH, also known as Hartmann-Shack) wavefront sensor and the Point-Diffraction Interferometer (PDI). 
The SH wavefront sensor has been widely used in the fields of Astronomy and Ophthalmic Optics due to its use in Adaptive Optics set-ups. The result is a really robust technology able to measure in real time the wavefront aberration.

On the other hand, the PDI is a tool not so extended as the SH, but with relevant applications in different fields. Moreover, in front of the lack of robustness, this technique presents the advantage that it is cheaper than the other and more versatile. In last decades, this instrument has been used in ophthalmic applications.

In this work we describe, analyze and compare both techniques in the analysis of imaging systems. The metrics used to compare both techniques are the Peak-to-Valley (PV) and the wavefront root-mean-square (RMS) error differences between the reconstructions of the wavefront obtained with both methods.

\section{Theoretical background}

As a general idea, geometrical aberration could be defined as the deviation that a wavefront experiments at the exit pupil (ExP) of an optical system respect to the ideal wavefront [2]. If such ideal wavefront is spherical, which means that there is not aberration, all the rays that came from the point object goes to the image point.

If a light ray travels a certain distance $s$ through a homogeneous medium of refractive index $n$ then, the optical path $L$ is defined as follows:

$$
L=n s
$$

If in its journey the ray passes through different media with different values of the refractive index $n_{i}$, travelling different distances $s_{i}$, then the optical path would be:

$$
L=\sum_{i} n_{i} s_{i}
$$

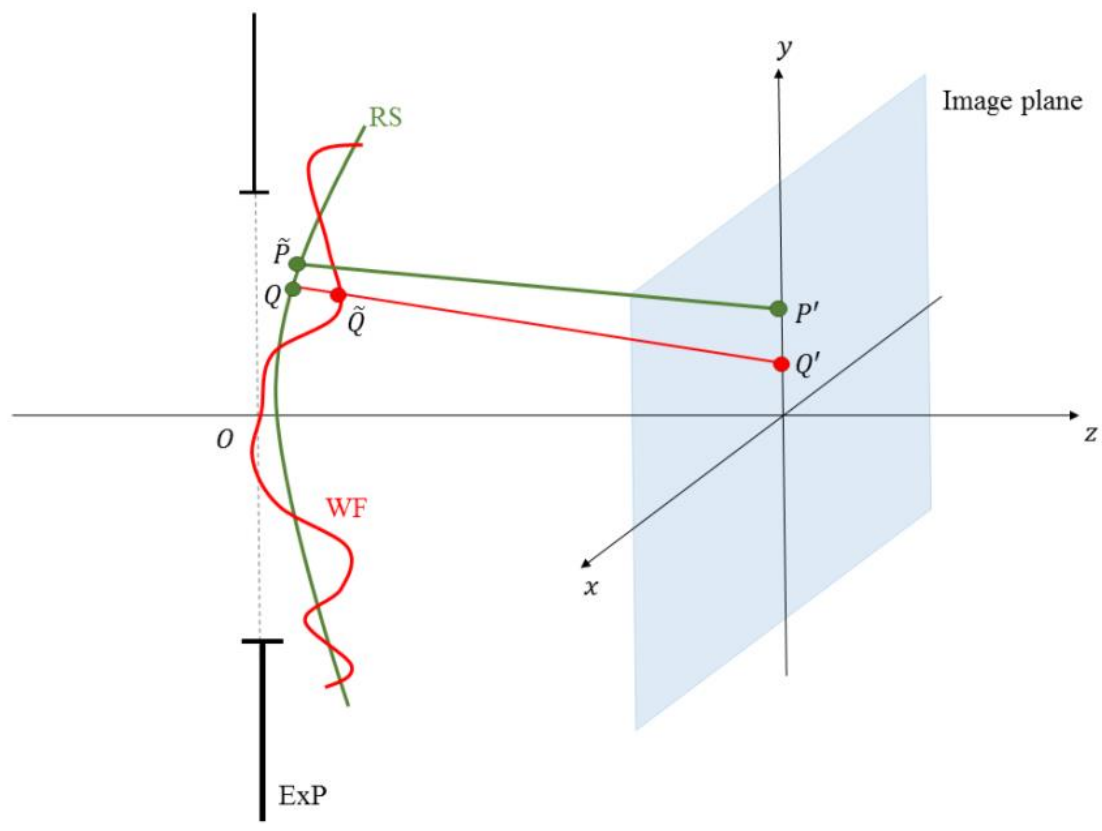

Figure 1. Basic scheme of an aberrated wavefront (which is the actual wavefront, WF) for an on-axis point object. $\tilde{P}$ is the point over the RS refered to the object point $P$, whereas $\widetilde{Q}$ is the point over the WF which corresponds to the ray that intercepts the RS at the point $Q$.

The most relevant ray from all of those which travels from the object point is the so-called chief ray (CR). If all the rays that are emerging from the object point are drawn in such a way that all of them present the same optical path of the CR, their tips generate a surface which is called the wavefront. In the case when this surface is spherical and presents its centre of curvature at the position of the paraxial or Gaussian image of the object point, the resulting image is perfect [1]. This essentially means that the optical path travelled by all the rays that emerge from a certain point $P$ (see Figure 1) and reach the corresponding image point $P^{\prime}$ is the same. Moreover, if the emerging wavefront deviates from the spherical one (which is also called 
the reference sphere, RS) then the image is aberrated. This means that rays do not travel the same optical path and their intersection with the paraxial plane is not at point $P^{\prime}$. The distance between the intersection points of each ray and the position of the paraxial image is the ray aberration. Thus, the wavefront aberration (WA) corresponding to a certain ray which intercepts the reference sphere at a certain point $Q$ (Figure 1), is defined as the optical path difference between the ray under consideration and the CR [3].

As is broadly explained in specific bibliography [1] [2] [3], it is possible to write the WA as a power series expansion. The interested reader can find a more extended, rigorous and specific derivation of that theory in [3].

\section{2.a. Zernike Polynomials}

The wavefront aberration of an optical system can be expressed as a function of a complete ${ }^{1}$ set of polynomials [3]. There are many sets of polynomials that fit this condition, but there is one broadly used in Optics which was introduced in 1934 by the Nobel Prize laureate in Physics, Fritz Zernike (1888 - 1966) [4].

Zernike polynomials are orthonormal and continuous over a unit circle. Since the majority of commonlyused optical systems present circular or spherical components and rotational symmetry, Zernike polynomials in polar coordinates $(x=\rho \cos \theta, y=\rho \sin \theta)$ are a good choice for describing wavefronts generated by those kind of systems.

As was mentioned before, in general, a function $W(\rho, \theta)$ which describes an arbitrary wavefront in polar coordinates $(\rho$ and $\theta)$, can be expressed in terms of a polynomial expansion as follows [5] [6]:

$$
W(\rho, \theta)=\sum_{n=0}^{\infty} \sum_{m=0}^{n} C_{n}^{m} Z_{n}^{m}(\rho, \theta)
$$

Where $C$ denotes the Zernike coefficient (also known as amplitude, Zernike term or "weight") which corresponds to a certain polynomial term $Z(\rho, \theta)$.

Since these polynomials are orthonormal in the unit circle, the normalization is chosen in the following way:

$$
N_{n}^{m}=\left[\frac{2(n+1)}{1+\delta_{m 0}}\right]^{1 / 2}
$$

where $\delta_{m 0}$ is the so-called Kronecker delta $\left(\delta_{m 0}=0 \text { for } m \neq 0\right)^{2}$.

For simplicity in the notation of those polynomials we can use Noll's sequential indices [6], in order to pass from $Z_{n}^{m}(\rho, \theta)$ to $Z_{j}(\rho, \theta)$. The basic rule for doing that is that the polynomial which presents an even value of $m$ obtains an even index $j$, and the other-way-round for the odd $m$ indices. Within a given $n$, lower values of $m$ obtain lower $j$ indices. For instance, the polynomial $Z_{2}^{-2}$ corresponds to $Z_{5}$, whereas $Z_{2}^{2}$ corresponds to the polynomial $Z_{6}$, according to Noll's notation.

As an illustrative example of the relation of Zernike polynomials with classical geometrical aberrations, Table 1 show few of those polynomials and its corresponding expression. A graphical representation is presented in Figure 2.

\footnotetext{
1 The term "complete" implies that any reasonably well-behaved function can be expanded as a series of functions of the set.

2 At that point, it is important to comment that two different nomenclatures are used in Zernike expansion for wavefront analys is. When the $N_{n}^{m}$ constant is included inside the $C_{n}^{m}$ coefficient, the expansion is known as Zernike Peak-to-Valley (PV) polynomials (also known as Wyant nomenclature), whereas when $N_{n}^{m}$ is explicitly included in the expression, the corresponding name is Zernike RMS polynomials or Noll's expansion [6]. Moreover, this different notation also includes a different ordination on the coefficients, meaning that it is really crucial to be consistent in the study with the notation used. This comment is necessary since the commercial software used in this work to obtain Zernike expansion from experimental data permits the use of both developments. In our study, Wyant nomenclature is used.
} 


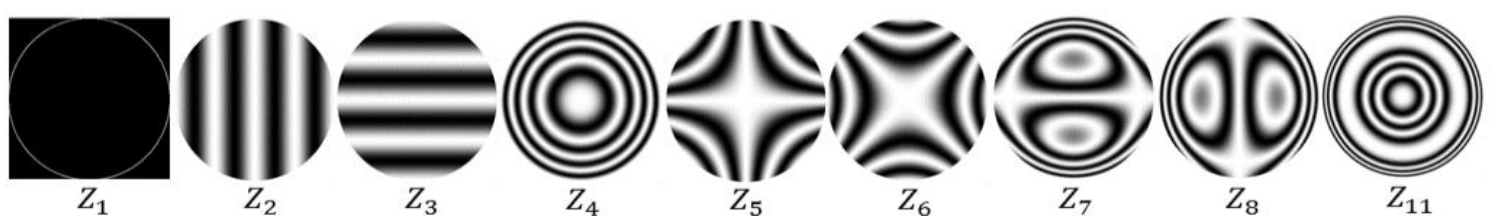

Figure 2. Pictorial representation of the wavefronts (i.e. phase maps) corresponding to aberrations presented in Table 1.

TABLE 1. Most known and relevant orthonormal Zernike circle polynomials and its corresponding aberrations.

\begin{tabular}{|c|c|c|c|c|c|c|}
\hline \hline$j$ & $n$ & $m$ & $N_{n}^{m}$ & Zernike Polynomials & $Z_{j}$ & Aberration name \\
\hline \hline 1 & 0 & 0 & 1 & 1 & $Z_{1}$ & Piston \\
2 & 1 & 1 & 2 & $\rho \cos \theta$ & $Z_{2}$ & Tilt in X direction \\
3 & 1 & -1 & 2 & $\rho \sin \theta$ & $Z_{3}$ & Tilt Y direction \\
4 & 2 & 0 & $\sqrt{3}$ & $2 \rho^{2}-1$ & $Z_{4}$ & Defocus \\
5 & 2 & -2 & $\sqrt{6}$ & $\rho^{2} \sin (2 \theta)$ & $Z_{5}$ & Astigmatism at 45o \\
6 & 2 & 2 & $\sqrt{6}$ & $\rho^{2} \cos (2 \theta)$ & $Z_{6}$ & Astigmatism at $0^{\circ}$ \\
7 & 3 & -1 & $\sqrt{8}$ & $\left(3 \rho^{3}-2 \rho\right) \sin \theta$ & $Z_{7}$ & Coma at 90 o \\
8 & 3 & 1 & $\sqrt{8}$ & $\left(3 \rho^{3}-2 \rho\right) \cos \theta$ & $Z_{8}$ & Coma at 0 o \\
11 & 4 & 0 & $\sqrt{5}$ & $6 \rho^{4}-6 \rho^{2}+1$ & $Z_{11}$ & Primary spherical aberration \\
\hline
\end{tabular}

Finally, a common metric of wavefront error is the RMS or wavefront error $\sigma$, which is defined in terms of the Zernike coefficients as follows [5]:

$$
\sigma=\left(\sum_{j=4}^{N} C_{j}^{2}\right)^{\frac{1}{2}}
$$

Where the first terms, which represent the aberrations of piston and tilts, are ignored. The reason for ignoring those terms is because they just provide information on system alignment.

\section{Description of the techniques}

\section{3.a. Shack-Hartmann wavefront sensor}

At the beginning of $20^{\text {th }}$ Century, researchers from the Astrophysical Institute of Postdam reported that the optics of their famous Great Refractor Telescope (an $80 \mathrm{~cm}$ refracting telescope) presented inadequate quality. German astrophysicist Johannes Hartmann (1865 - 1936) proposed to set a screen with a series of holes in it, at the aperture of the telescope. With this configuration, Hartmann was able to test the optical quality of the telescope by using the spot diagram generated with this mask.

In late 1960s, the Optical Science Center (OSC) at the University of Arizona by the US Air Force, pointed out a technical problem: Earth's atmosphere limits the image quality and exposure time in some astronomical observations from terrestrial observatories. Dr. Roland Shack (Chicago, Illinois, USA, 15 th January 1927) found the solution: he took the classical Hartmann test and replaced the array of holes used at that time by an array of lenses [7]. Then, the Shack-Hartmann (SH) (sometimes called Hartmann-Shack) wavefront sensor device was born.

Nowadays, a common SH sensor basically comprises a microlens array and a CCD camera. The wavefront which is tended to be measured is divided by the microlens array in such a way that each light division is brought to a particular location in the focal plane of the array. If the wavefront is aberrated, these spots shift from the regularly spaced ideal spot pattern produced by a plane wavefront (see Figure 3). These 
displacements provide information on the local slope of the wavefront over each microlens, and then the WA can be reconstructed using an indicated software.

Although originally designed to be used in correction atmosphere turbulence in optical Astronomy [8], during the last past two decades SH sensors have successfully been used in other fields such as the characterization of ophthalmic lenses, analysis and compensation of ocular aberrations [9], or the generation of optical tweezers [10] and the correction of its aberrations [11]. For those reasons, a SH wavefront sensor seems to be a powerful tool in order to analyze the quality of optical instruments.
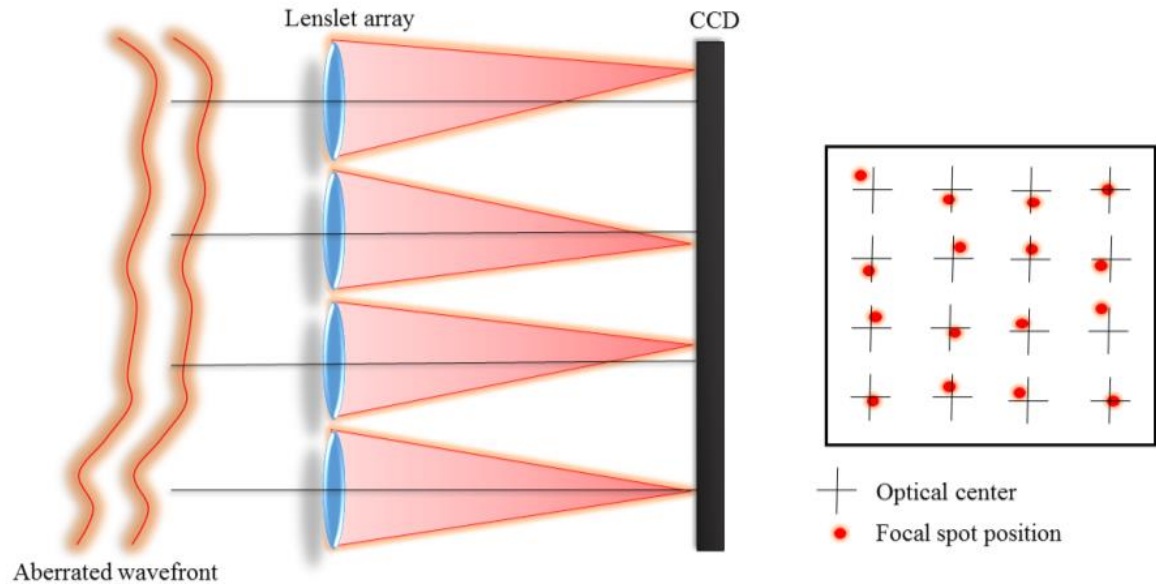

Aberrated wavefront

Figure 3. Schematic of a SH wavefront sensor working when an aberrated wavefront reaches the microlens array.

\section{3.b. Point-Diffraction Interferometer}

In 1933, a new kind of simple interferometer for measuring phase variations across a wavefront was introduced [12]: the Point-Diffraction Interferometer (PDI). Furthermore, in early 1970s, R. N. Smartt, from University of Massachusetts, reintroduced the instrument and set its potential applications [13]. This instrument is the Point-Diffraction Interferometer (PDI), which is a simple common-path interferometer used to directly measure optical path differences. A PDI basically consists on a semitransparent plate with a clear pinhole, as shown in Figure 4.

When a light beam reaches the plate, a spherical reference wave is produced by diffraction at the clear pinhole while the rest of the beam passes through the plate without any change in phase. If the size of the pinhole and the transmittance of the plate are chosen in such a way that both beams have similar amplitude, well contrasted fringes will be observed in any plane placed after the plate. Figure 4 illustrates the basis of this technique.

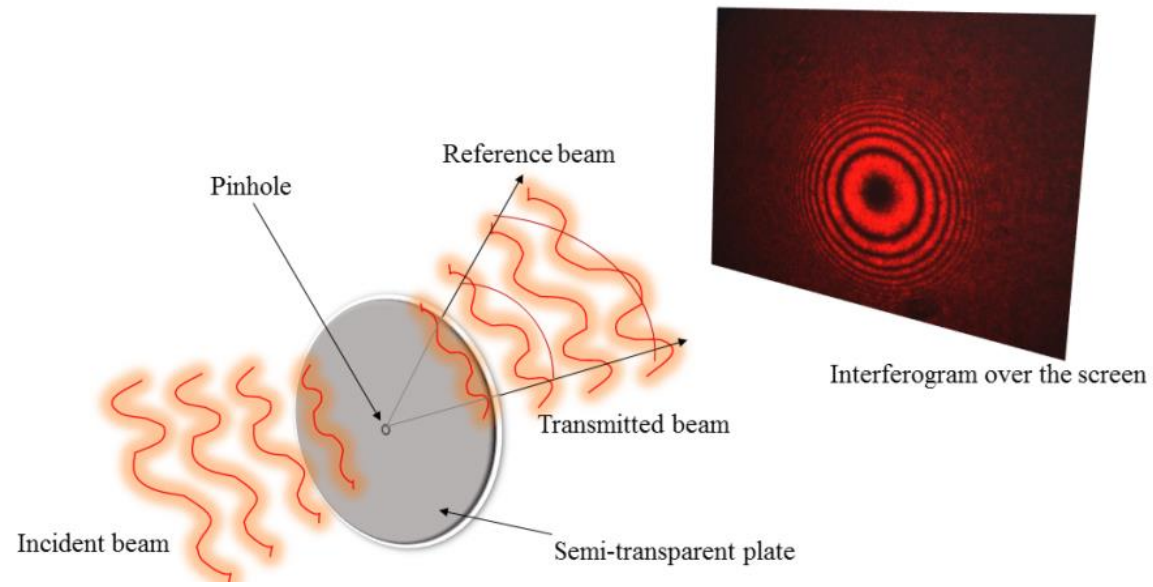

Figure 4. Basic principle of PDI. 
In recent years, this instrument has been taking some relevant interest in the field of Ophthalmic Optics for the characterization of progressive lenses [14]. Since progressive lenses generate complex wavefronts [15] and PDI is capable to analyse them, this technique seems to be a powerful and relevant tool in the study of noisy beams such the ones generated by biological samples. In this direction, relevant implementations has been reported in the analysis of fish lenses [16].

\section{Experimental set-up}

Our goal is to see the equivalence of results between both techniques. Thus, in order to do that, maintaining the same conditions of measurements using both techniques is necessary. For this reason, a dual set-up should be designed taking into account the differences of measurement configurations of both techniques.

In order to perform the experiments, the best measuring configuration is to send plane waves to the system under test. In our set-up, an achromatic doublet of $500 \mathrm{~mm}$ of focal length is used as a collimator for this purpose.

In our set-up, a conjugation lens is required in order to bring the aberrated wavefront to the SH entrance pupil. Furthermore, this system needs to provide a total magnification in such a way all the measuring area enters in the sensors surface, otherwise we will be "cutting" the wavefront and therefore losing some information.

According to Abbe - Rayleigh image formation theory [17] [18], all the aberrating and diffracting effects of the optical system are considered at its ExP. Thus, this conjugation lens has another function: to bring the image of the ExP to the microlenses array plane. Considering this requirements, a photographic objective was chosen as a conjugating system (CL). A He-Ne laser $(\lambda=633 \mathrm{~nm})$ was used in all the experiments as a light source.

Different systems were analysed with this set-up. The semitransparent plate of the PDI was made by coating a glass substrate with $\mathrm{Cr}$. The optical density was 2.5 and the pinhole diameter was $15 \mu \mathrm{m}$. This plate was mounted over a $x y z$ support.

Finally, the SH device used in this work was a HASO32TM from the Imagine Optics Company. The lenslet array consists on $32 \times 32$ microlenses and a 512x512 pixels CCD sensor of $5 \times 5 \mathrm{~mm}$ area.

Considering all those requirements, the set-up shown in Figure 6 was mounted in an optical bench. When measurements are done, first the SH configuration (see Figure $6 \mathrm{a}$ )) is used and then, the wavefront sensor is removed and replaced by a simple CCD camera and the PDI is placed near the focus region of the system under test (see Figure 6 b)).
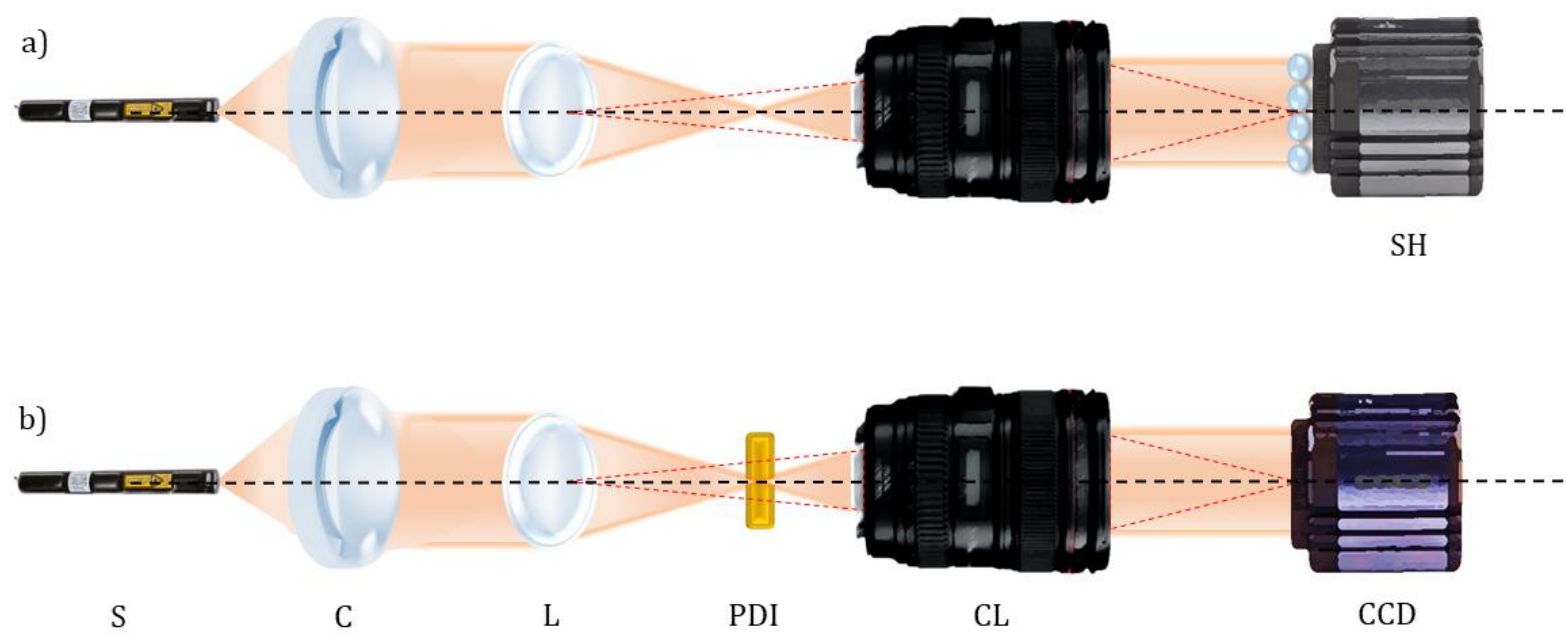

Figure 6. Scheme of the set-up designed and used in the laboratory. Light travels from left to right, and S represents the light source (in this case an He-Ne laser), C the collimating lens, L the optical system to be analysed (which in this case was a single biconvex lens), and CL represents the conjugation lens. a) is the configuration for measurements using SH wavefront sensor and b) for PDI analysis. Red-dotted lines show the formation of the image of the wavefront of the ExP over the measurement plane. 
A software for computing Zernike coefficients is supported by the manufacturer for the SH device used in this study. For the computation of those coefficients from the interferogram the Atmosfringe® software [19] was used.

\section{Results}

Using the set-up described in previous section, different systems were analysed (see Table 2).

TABLE 2. Lens analysed

\begin{tabular}{|c|c|c|}
\hline \hline System & Manufacturer & Focal length (mm) \\
\hline \hline Biconvex & Unknown & 200 \\
LE1202 & ThorLabs & 200 \\
LE1157 & ThorLabs & 250 \\
\hline
\end{tabular}

Because tilts and also defocus aberrations just provide information on the system misalignment, those coefficients were always excluded. Moreover, since the systems under test are conventional lenses, just the coefficients up to $11^{\text {th }}$ order were considered, because higher orders are null for this kind of systems, i.e. they are not expected to present high-order aberrations such as trefoil. Otherwise, including more coefficients in the development will imply adjusting noise [20].

For the measurements carried out with the different systems under test, results shown in following figures were obtained, where pictorial representations of the wavefronts are shown. A visual inspection shows that both techniques provide quite similar results. These images were obtained implementing equation (3) in a MATLAB $®$ code. For each data set, the $\sigma$ and PV values were computed.

The maximum RMS difference between both techniques obtained for those systems is about $0.01 \lambda$ and the maximum PV difference is $\lambda / 10$.
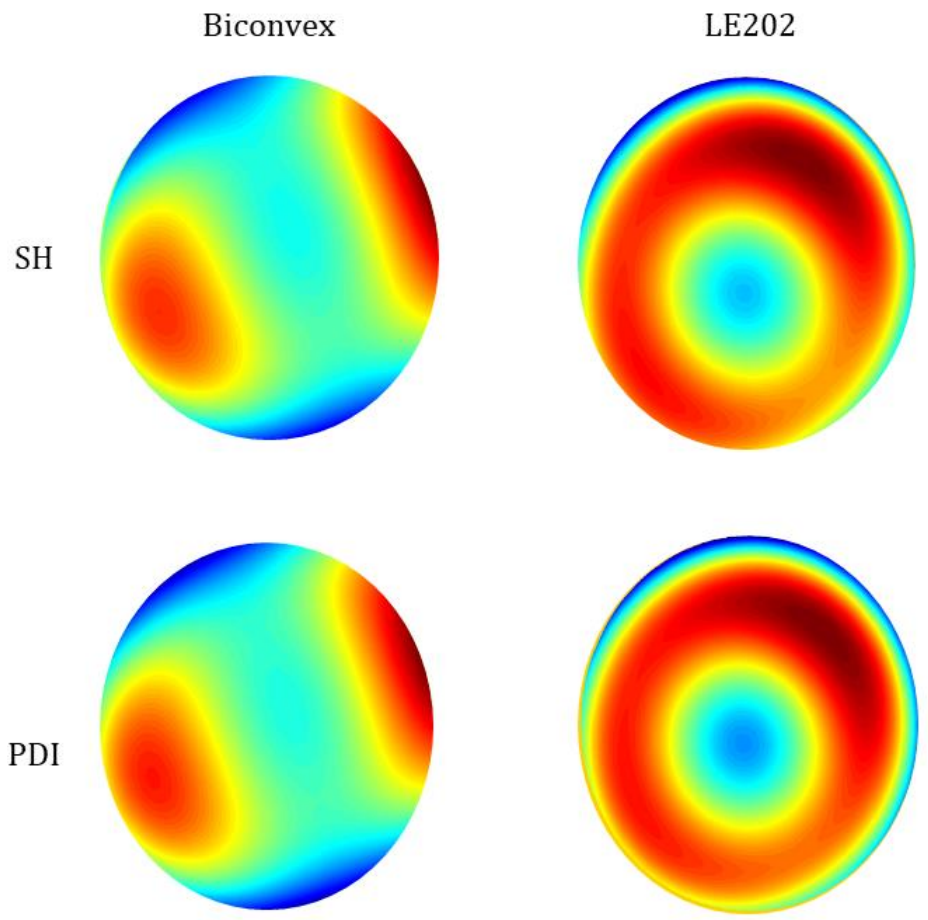

LE1157
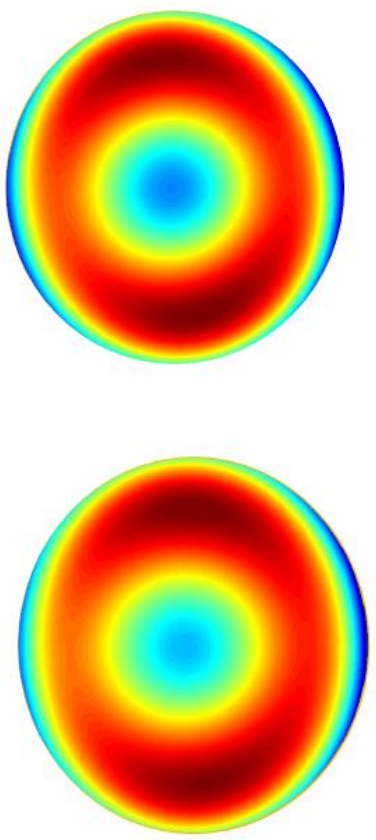

Figure 7. WAs computed in MATLAB $®$ with SH and PDI data, for the three lenses under study (rows correspond to the techniques and columns to the systems analysed). Tilt and defocus were always excluded. 
ÓPTICA PURA Y APLICADA

www.sedoptica.es

TABLE 3. RMS and PV differences between both techniques

\begin{tabular}{|c|c|c|}
\hline \hline System & $\Delta \sigma(\lambda)$ & $\Delta \mathrm{PV}(\lambda)$ \\
\hline \hline Biconvex & 0.001 & 0.010 \\
LE1202 & 0.005 & 0.017 \\
LE1157 & 0.001 & 0.082 \\
\hline
\end{tabular}
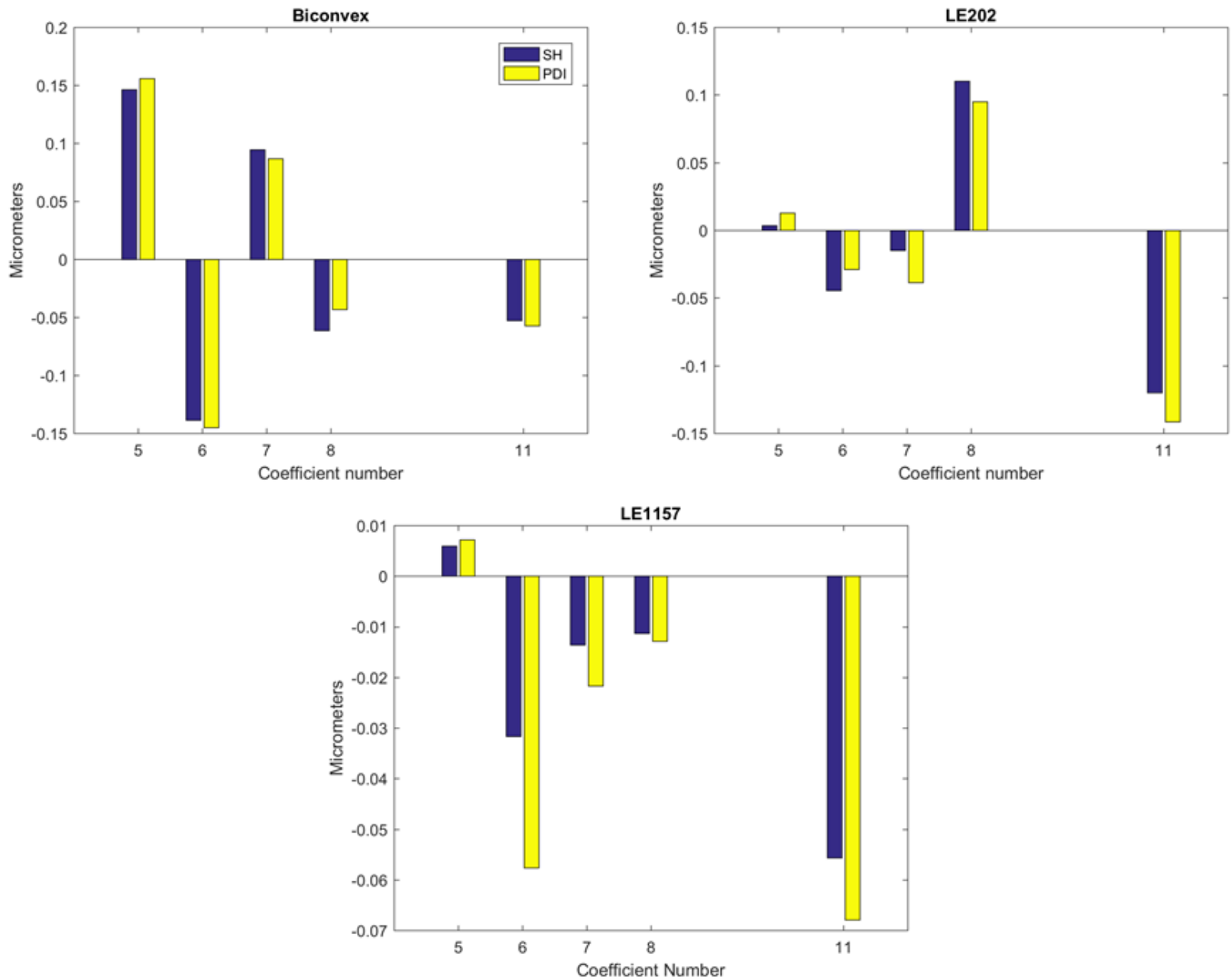

Figure 8. Values in micrometers of the coefficients for each system obtained using both techniques. Tilt and defocus were always excluded.

\section{Discussion}

Equivalence of results between SH and PDI was experimentally proved with a difference in the RMS between both techniques of $0.01 \lambda(0.01 \mu \mathrm{m})$. This equivalence was previously reported by Bueno et al. [21] for the case of phase plates obtaining a maximum difference in the RMS of $\lambda / 10$. As far as we know, no-one has presented or reported this equivalence in the case of real imaging systems as it was done in this work.

As was briefly commented in each method section, both techniques used in this work are operationally different, and each one presents advantages and disadvantages respect the other.

On the one hand, PDI is a very simple technique due to the fact that it is composed by one simple optical element. The interference pattern provided by PDI can be easily interpreted as the phase aberration of the 
wave, plus some small amount of defocus and tilt in case the pinhole is not correctly placed at focus. This technique permits the direct visualization of the constant phase fringes. However, the computation of the WA requires post-processing of the images from manual tracking dark fringes using [19], with the accuracy limitations that a manual procedure implies.

On the other hand, SH wavefront sensor is a well-known, widely and commonly used device in ocular, astronomical and optical testing applications. Unlike the PDI, the analysis to obtain WA can be done in real time, which allows easy experimental alignment. Moreover, this sensor does not precise monochromatic light for measuring, but presents the drawback that details smaller than microlenses size are not resolved or detected. Development of a dual compact set-up combining both techniques seems to be able to provide a robust design.

\section{Conclusions}

We have demonstrated the equivalence of two different and complementary techniques (PDI and SH sensor) for measuring the WA of imaging optical systems such as single lenses under the same experimental conditions when first few Zernike coefficients are taken into account. Under the conditions of our experiments, results provide the same information within the experimental error. In different applications, the combined use of both techniques, could offer the optimum solution, by merging high resolution from the PDI with the robustness of SH.

Since there are commercial devices for characterization of progressive addition lenses based on SH sensors [22] and the PDI has been shown as a potential technique for characterizing those systems [15] we are interested in the development of an integrated set-up which includes both techniques. Some experiments were carried out at the end of this study in that direction, and what was observed is that this kind of spectacle lenses need a mapping system for the measurements. Furthermore, we are also interested in the development of a dual set-up for the characterization of Intraocular lenses (IOLs) using both SH and PDI. Preliminarily works [23] presented interesting designs for characterizing these systems.

In conclusion, future works may combine both techniques in the characterization of different kinds of ophthalmic systems, taking advantage of the two methods.

\section{Acknowledgements}

The authors acknowledge the 'Agencia Estatal de Investigación' (AEI) and the 'Fondo Europeo de Desarrollo Regional' (FEDER), under project FIS2016-77319-C2-2-R of the Spanish 'Ministerio de Economía, Industria y Competitividad'.

The corresponding author wants to express his gratitude to Dr. Vallmitjana and Dr. Bosch for their patience and supervision of the present work, and thanks to Dr. Justo Arines from USC for his helpful comments. Also he wants to thank all the people from the Optics and Photonics Research Group for their help and kindness during his stage. This work is the result of the MSc degree Thesis of the corresponding author defended on September 2017 within the Master in Photonics taught by the Universitat Politècnica de Catalunya (UPC), the Universitat de Barcelona (UB), the Universitat Autònoma de Barcelona (UAB) and the Institute of Photonics Sciences (ICFO). 